\title{
Aspirants entrepreneurs : Le cas de la génération Y
}

\author{
Maripier Tremblay, Josée Audet et Yvon Gasse \\ Chaire en entrepreneuriat et innovation \\ Université Laval
}

\section{INTRODUCTION}

\section{Concilier la famille et le travail, protéger l'environnement, respecter les différences et s'impliquer dans la communauté sont quelques exemples de valeurs partagées par les personnes de la génération $Y$, les distinguant de leurs prédécesseurs.}

La relève d'entreprise constitue aujourd'hui un défi de taille dans plusieurs pays, alors que le nombre d'entrepreneurs potentiels apparaît inférieur au nombre d'entrepreneurs sur le point de prendre leur retraite. Parmi le bassin d'entrepreneurs potentiels, les jeunes de la génération $\mathrm{Y}$ (aujourd'hui dans la vingtaine) occuperont vraisemblablement une place prépondérante. Par ailleurs, les Y représentent aujourd'hui un sujet d'actualité récurrent. Leurs particularités en matière de valeurs, de motivations et de fonctionnement, engendrent certains défis pour les gestionnaires et les responsables de ressources humaines. En effet, plusieurs travaux montrent que les membres de cette génération ont des valeurs et des comportements très différents de leurs prédécesseurs, particulièrement sur le marché du travail ${ }^{1}$. Concilier la famille et le travail, protéger l'environnement, respecter les différences et s'impliquer dans la communauté sont quelques exemples de valeurs partagées par les personnes de la génération $\mathrm{Y}$, les distinguant de leurs prédécesseurs.

Or, ces mêmes distinctions laissent envisager que les aspirants entrepreneurs de cette nouvelle génération pourraient ne pas posséder les mêmes motivations et les mêmes objectifs entrepreneuriaux que les générations précédentes. On peut se demander si ces caractéristiques se reflèteront dans le profil entrepreneurial de ces derniers. Ainsi, quelles sont leurs motivations à entreprendre et quels modèles d'affaires privilégient-ils? Comme ils constituent la relève entrepreneuriale et qu'une partie du développement économique futur repose sur leur succès, de telles connaissances permettraient de mieux les préparer et les guider dans leurs efforts entrepreneuriaux.

Bien que les caractéristiques de cette jeune génération de même que les enjeux que représentent leur arrivée sur le marché du travail soient de plus en plus documentés, peu de travaux ont encore abordé l'entrepreneuriat chez les Y. Un certain nombre d'écrits traitent des caractéristiques des $\mathrm{Y}$ susceptibles d'affecter leurs comportements en milieu de travail, principalement dans un rôle d'employé ${ }^{2}$. Cependant, la littérature abordant leur qualité d'entrepreneur est pratiquement inexistante, vu la récente émergence du phénomène. Conséquemment, il a été décidé de mener une étude de type exploratoire sur le sujet, laquelle vise à dresser un portrait des aspirants-entrepreneurs de la génération $\mathrm{Y}$ et à dégager des éléments pouvant les caractériser et possiblement les distinguer des entrepreneurs des générations antérieures.

La première partie de cette étude est consacrée à la revue de la littérature portant sur les principales caractéristiques des membres de la génération Y. Par la suite, les objectifs de l'étude ainsi que la méthodologie seront détaillés. Finalement, les caractéristiques de l'échantillon retenu seront présentées de même que les constats intéressants tirés de l'analyse, avant de conclure sur les suites à donner à cette étude. 


\section{QUI SONT LES Y ?}

Bien que les chercheurs ne s'entendent pas tous pour délimiter les années exactes entourant chaque génération, on associe généralement la génération Y aux jeunes nés entre les années 1978 et 1990. Grâce à leur grand nombre, les $\mathrm{Y}$ auront un poids et une influence non négligeables dans le monde des affaires; ils sont destinés à «brasser» les pratiques courantes de gestion et à avoir un effet positif sur les organisations ${ }^{3}$. Aux États-Unis notamment, cette génération serait la plus diversifiée, avec plus du tiers de ses membres provenant d'une ethnie autre que blanche non hispanique ${ }^{4}$. Cette génération d'environ 80 millions d'individus serait la plus grande, la plus éduquée et la plus diversifiée de l'histoire américaine ${ }^{5}$. Ce sont ceux qui voyagent le plus et qui sont les plus confortables avec la technologie. Ils considèrent que leur contribution à la société ainsi que leur rôle en tant que parents sont plus importants qu'un gros salaire ${ }^{6}$. Au Québec, les Y seraient au nombre de 1317839 , soit environ $17 \%$ de la population ${ }^{7}$.

\subsection{Les Y en tant que citoyens}

Les Y sont réputés être optimistes, portés vers l'accomplissement, faire montre d'une grande estime de soi, avoir des habiletés sociales et prôner l'éthique et la diversité.

La génération Y a grandi avec la technologie. Les jeunes de cette génération sont non seulement à l'aise avec cet élément, mais y accorde une grande importance ${ }^{8}$. On s'entend également pour dire que les Y sont très attachés à leurs parents. Même pour ceux qui ont quitté le nid familial, le niveau de dépendance envers leurs parents demeure élevé, surtout au niveau financier ${ }^{9}$. Les $\mathrm{Y}$ sont réputés être optimistes, portés vers l'accomplissement, faire montre d'une grande estime de soi, avoir des habiletés sociales et prôner l'éthique et la diversité. Les membres de la génération Y (ou la génération «moi ») sont aussi plus prédisposés que leurs parents à abandonner l'école ou du moins à s'accorder du temps pour voyager ou accepter des emplois non reliés à une carrière. En général, ils attendront plus longtemps que leurs parents pour acheter une maison, se marier ou avoir des enfants ${ }^{10}$. Les $Y$ considèrent que les gens, les relations et les styles de vie sont les composantes clés du bonheur, et ils ne sont pas enclins à compromettre ces aspects pour leur carrière ${ }^{11}$. Les individus appartenant à cette génération sont idéalistes et possèdent de grandes attentes. Ils aiment avoir un mentor et ils veulent apprendre des autres $^{12}$.

\subsection{Les Y en tant qu'employés}

\section{Les employés de type $Y$ recherchent un emploi orienté vers le travail d'équipe et sur le « multitasking », offrant un environnement structuré. L'action collective est privilégiée, ainsi que la responsabilisation et la participation dans les prises de décisions.}

Une part importante des attentes des Y concerne leur environnement professionnel. Les employés de type $\mathrm{Y}$ recherchent un emploi orienté vers le travail d'équipe et sur le «multitasking», offrant un environnement structuré. L'action collective est privilégiée, ainsi que la responsabilisation et la participation dans les prises de décisions $^{8}$. Les Y valorisent l'éducation et sont attirés par des organisations socialement responsables encourageant le coaching au travail, leur permettant à la fois de développer des aptitudes nouvelles et de les enseigner aux autres ${ }^{11}$. Ils recherchent également la reconnaissance et le respect et souhaitent établir une bonne relation avec leur patron ${ }^{13}$. Les $\mathrm{Y}$ recherchent le feedback et s'attendent à des gratifications instantanées de la part de leur employeur; ils ont besoin d'être rassurés et guidés dans l'accomplissement de leur travail ${ }^{6}$.

Les Y ont aussi des attentes élevées envers euxmêmes. Cherchant constamment à identifier et résoudre les problèmes, ils ont besoin de se dépasser. Les membres de cette génération valorisent le développement d'aptitudes particulières de travail, et ils apprécient les défis offerts par de nouvelles opportunités. Capables de travailler efficacement et rapidement, il leur faut cependant plus que des récompenses floues ${ }^{14}$. D'ailleurs, le fait qu'ils s'adaptent facilement aux changements, notamment technologiques, et qu'ils valorisent le mentorat et la formation, leur permet de demeurer à jour et de pouvoir changer 
d'organisation facilement s'ils le désirent ${ }^{1}$. En outre, les formes de motivation traditionnelles telles que les promotions, les bonus et les paies accordées selon le mérite rejoignent peu les $\mathrm{Y}^{3}$.

\subsection{Les Y en tant qu'entrepreneurs}

\section{Comme leurs attentes professionnelles sont élevées et que les employeurs n'arrivent que partiellement à les combler, les $\mathbf{Y}$ envisagent devenir leur propre patron.}

Les jeunes de la génération $\mathrm{Y}$ font montre d'un esprit entrepreneurial. Ils pensent comme des entrepreneurs, mais aussi comme des consommateurs. Leur jeunesse et leur énergie sont des avantages. Cependant, en contrepartie, ils sont encore un peu naïfs face au monde entrepreneurial $^{14}$. Les $\mathrm{Y}$ ont à cœur l'équilibre entre le travail et la famille, ce que leurs parents n'ont généralement pas pu leur offrir. La voie entrepreneuriale devient pour eux une façon de résoudre ce problème. Comme leurs attentes professionnelles sont élevées et que les employeurs n'arrivent que partiellement à les combler, les Y envisagent devenir leur propre patron.

\section{CADRE CONCEPTUEL ET MÉTHODOLOGIE}

Considérant le nombre restreint de travaux effectués sur le sujet, le recours à un cadre conceptuel rigide n'était pas souhaitable. Une démarche fondamentalement exploratoire s'avérait plutôt nécessaire pour dresser un portrait d'aspirants entrepreneurs de la génération $\mathrm{Y}$ et dégager des éléments pouvant les caractériser et possiblement les distinguer des générations antérieures. Pour ce faire, nous avons procédé à une analyse de contenu de 59 demandes de bourses présentées entre 2006 et 2009 par des étudiants du premier cycle de l'Université Laval dans le cadre du profil entrepreneurial $^{18}$.

Le formulaire de demande de bourse comprenait trois sections comportant majoritairement des questions ouvertes. La première section visait à mieux identifier l'étudiant par le biais de données sociodémographiques: âge, genre, programme d'études, etc. Une deuxième section consistait en une question portant sur les expériences à caractère entrepreneurial des étudiants, soit celles où ils
D'ailleurs, la génération $\mathrm{Y}$ serait trois fois plus encline à avoir démarré une entreprise en sortant de l'école que les Baby Boomers : $27 \%$ contre $9 \%{ }^{15}$. Il semble également que $36 \%$ des $\mathrm{Y}$ aient déjà été travailleurs autonomes ou aient déjà possédé une entreprise. De plus, les aspirants entrepreneurs seraient à $54 \%$ des hommes et à $46 \%$ des femmes. Il semble que les $\mathrm{Y}$ ont tendance à démarrer une entreprise très tôt dans leur cheminement. Bien qu'ils échouent parfois rapidement, ils apprennent beaucoup et continuent d'avancer. D'autant plus qu'ils sont confortables avec un monde d'affaires en constant changement ${ }^{5}$. Par ailleurs, les $\mathrm{Y}$ tendent à démarrer leur entreprise avec des partenaires, car ils comprennent intuitivement le pouvoir de la collaboration et de l'apprentissage collectif. Ils ont aussi tendance à vouloir accomplir une sorte de mission sociale. Si la plupart des entrepreneurs attendent que leur compagnie soit établie et profitable, les jeunes entrepreneurs ont plutôt tendance à s'impliquer tout de suite dans une mission sociale $^{16}$. Aussi, la nouvelle génération serait beaucoup plus interpellée par les problèmes environnementaux que ses aînés ${ }^{17}$. avaient eu à faire preuve de leadership et d'initiative. Finalement, la dernière section reposait sur une question ouverte où l'étudiant était invité à décrire son projet entrepreneurial et la contribution qu'il comptait apporter à son développement.

\subsection{Dimensions étudiées}

Les dimensions retenues pour être examinées plus en profondeur ont été sélectionnées à la suite d'une première analyse d'un échantillon de dossiers. Le choix des dimensions s'est fait sur la base des caractéristiques des Y répertoriées dans la littérature de même qu'en tenant compte des facteurs généralement reconnus comme étant associés à la propension à entreprendre. Les variables retenues sont regroupées en trois catégories: les variables socio-démographiques et les antécédents, les aspirations entrepreneuriales ainsi que le projet d'entreprise. Il est important de mentionner que pour l'ensemble des variables retenues, il a parfois été impossible de retracer l'information dans le texte présenté par l'étudiant. 


\section{RÉSULTATS}

\subsection{Portrait sociodémographique et antécédents}

L'échantillon est composé d'une population de cinquante-neuf (59) étudiants du premier cycle universitaire, inscrits au profil entrepreneurial de l'Université Laval entre les années 2006 et 2009. Les étudiants sélectionnés étaient âgés entre 22 et 29 ans, ce qui correspond aux balises d'âge généralement comprises à l'intérieur de la génération Y. La moyenne d'âge de l'échantillon est de 25 ans, un échantillon est constitué de 54,2\% d'hommes. Les étudiants provenaient de domaines d'études variés: un bon nombre d'entre eux étudient en administration des affaires (30,5\%), d'autres en aménagement, architecture et les arts visuels $(20,3 \%)$, agriculture et alimentation $(13,6 \%)$ ou encore les lettres $(10,2 \%)$. On en retrouve aussi, mais dans une plus faible proportion, en sciences et génie $(10,2 \%)$, médecine $(3,4 \%)$, foresterie, géographie et géomatique $(1,7 \%)$ ainsi qu'en musique $(1,7 \%)$. Certains étudiants possèdent un parent entrepreneur $(27,1 \%)$. De plus, $11,9 \%$ des étudiants ont mentionné dans leur demande de bourse qu'ils avaient bénéficié d'une éducation scolaire axée sur la participation à des projets ou à des programmes scolaires spécialisés. Par ailleurs, il est pertinent de mentionner qu'une majorité d'étudiants de cette étude ont déjà accompli des activités témoignant d'un certain leadership (74,6\%), notamment par l'organisation d'événements et la gestion de projets $(28,8 \%)$. Finalement, presque la moitié d'entre eux $(45,8 \%)$ ont effectué des voyages ou des échanges à l'étranger. Le tableau 1 présente un récapitulatif des variables socio-démographiques et des antécédents.

Tableau 1 : Variables socio-démographiques et antécédents

\begin{tabular}{|c|c|c|}
\hline & $\mathbf{N}$ & $\%$ \\
\hline$\hat{\text { Age }}$ & & \\
\hline Entre 22 et 23 ans & 22 & 40 \\
\hline Entre 24 et 26 ans & 21 & 38,2 \\
\hline Entre 27 et 29 ans & 12 & 21,8 \\
\hline Moyenne d'âge & \multicolumn{2}{|c|}{25 ans } \\
\hline \multicolumn{3}{|l|}{ Genre } \\
\hline Homme & 32 & 54,2 \\
\hline Femme & 27 & 45,8 \\
\hline \multicolumn{3}{|l|}{ Formation } \\
\hline Administration des affaires & 18 & 30,5 \\
\hline Sciences et génie & 6 & 10,2 \\
\hline Agriculture et alimentation & 8 & 13,6 \\
\hline Aménagement, architecture et arts visuels & 12 & 20,3 \\
\hline Foresterie, géographie et géomatique & 1 & 1,7 \\
\hline Lettres & 6 & 10,2 \\
\hline Médecine & 2 & 3,4 \\
\hline Musique & 1 & 1,7 \\
\hline \multirow[t]{2}{*}{ Autres } & 5 & 8,5 \\
\hline & $\mathbf{N}$ & $\%$ \\
\hline \multicolumn{3}{|l|}{ Parent(s) entrepreneur(s) } \\
\hline$O u i$ & 16 & 27,1 \\
\hline Non & 1 & 1,7 \\
\hline$N / D^{*}$ & 42 & 71,2 \\
\hline \multicolumn{3}{|l|}{ Formation « par projets » } \\
\hline$O u i$ & 7 & 11,9 \\
\hline$N / D^{*}$ & 52 & 88,1 \\
\hline \multicolumn{3}{|l|}{$\begin{array}{l}\text { Activités de leadership } \\
\end{array}$} \\
\hline Président de classe, d'association ou de comité & 11 & 18,6 \\
\hline Capitaine d'une équipe sportive & 9 & 15,3 \\
\hline Organisation d'événements, gestion de projets & 17 & 28,8 \\
\hline Emploi ou fonction professionnelle & 4 & 6,8 \\
\hline Autres & 3 & 5,1 \\
\hline$N / D^{*}$ & 15 & 25,4 \\
\hline \multicolumn{3}{|l|}{ Voyages/échanges } \\
\hline Oui & 27 & 45,8 \\
\hline$N / D *$ & 32 & 54,2 \\
\hline
\end{tabular}

*Information non disponible 


\subsection{Les aspirations entrepreneuriales}

En ce qui concerne les motivations des étudiants à créer leur entreprise, plusieurs (31 \%) semblent poussés par un besoin d'accomplissement ou de réalisation de soi, alors que près de $17 \%$ sont animés par un besoin d'autonomie et de liberté, c'est-àdire le besoin d'être indépendant et libre de prendre ses propres décisions, de ne pas être subordonné à un supérieur.

Les variables à l'étude concernaient les expériences qui avaient façonné le caractère entrepreneurial des étudiants et leurs motivations à se lancer en affaires. En ce qui a trait aux différentes expériences, on remarque que plus de la moitié des étudiants a été impliquée dans des activités de nature sociale, communautaire ou environnementale (58\%). Également, $53 \%$ ont mentionné avoir eu un emploi ou un stage dans un commerce, alors que $41 \%$ ont affirmé avoir été impliqués dans le domaine sportif. On remarque finalement que $30 \%$ des étudiants de l'échantillon ont créé une entreprise ou ont été travailleurs autonomes, et $20 \%$ ont effectué de la gestion de projets ou de l'organisation d'événements.

En ce qui concerne les motivations des étudiants à créer leur entreprise, plusieurs (31\%) semblent poussés par un besoin d'accomplissement ou de réalisation de soi, alors que près de $17 \%$ sont animés par un besoin d'autonomie et de liberté, c'est-à-dire le besoin d'être indépendant et libre de prendre ses propres décisions, de ne pas être subordonné à un supérieur. D'autres étudiants souhaitent aussi poursuivre leur passion (15\%), être reconnus dans leur secteur d'activités (3\%), accéder à un certain pouvoir $(7 \%)$ ou relever des défis $(8 \%)$. Le tableau 2 résume le profil entrepreneurial de l'échantillon.

Tableau 2 : Aspirations entrepreneuriales

\begin{tabular}{r|cc}
\hline & $\mathbf{N}$ & $(\boldsymbol{\%})$ \\
\hline Expériences & & \\
\hline Création d'entreprise ou travailleur autonome & 13 & 30 \\
Gestion de projets, organisation d'événements & 14 & 20 \\
Emploi ou stage & 39 & 53 \\
Implication domaine sportif & 25 & 41 \\
Implication sociale, communautaire ou & & 58 \\
environnementale & 34 & 32 \\
Autres & 19 &
\end{tabular}

\begin{tabular}{r|rr}
\hline Motivations & $\mathbf{N}$ & \% \\
\hline Besoin d'accomplissement & 17 & 31 \\
Besoin d'autonomie et de liberté & 10 & 17 \\
Besoin de pouvoir & 4 & 7 \\
Besoin de défis/ambition & 5 & 8 \\
Besoin de reconnaissance & 2 & 3 \\
Poursuivre sa passion & 9 & 15 \\
Non disponible & 11 & 19 \\
\hline
\end{tabular}




\subsection{Le projet entrepreneurial}

La plupart des projets envisagés par $63 \%$ des répondants déboucheront sur la mise sur pieds d'une entreprise, bien que $20 \%$ considèrent devenir travailleurs autonomes. Par ailleurs, $6 \%$ des étudiants ont un projet d'organisme à but non lucratif.

Une autre catégorie de variables se rapportait plus spécifiquement au projet d'entreprise des étudiants. En ce qui concerne les types de projets envisagés, les entreprises de service sont les plus prisées $(37 \%)$, suivies des commerces $(17 \%)$. De plus, quelques étudiants envisagent de démarrer leur entreprise dans le secteur agroalimentaire $(12 \%)$. Les projets les plus rares sont de nature technologique $(5 \%)$, touristique $(2 \%)$ ou artisanale $(2 \%)$.

La plupart des projets envisagés par $63 \%$ des répondants déboucheront sur la mise sur pieds d'une entreprise, bien que $20 \%$ considèrent devenir travailleurs autonomes. Par ailleurs, $6 \%$ des étudiants ont un projet d'organisme à but non lucratif telles que des organismes de charité ou des centres de formation et près de $5 \%$ une coopérative. Finalement, une plus faible proportion des projets sont de nature événementielle $(2 \%)$.

En ce qui a trait aux lieux géographiques considérés par les étudiants, $37 \%$ envisagent la création de leur entreprise en milieu urbain ${ }^{\mathrm{a}}$. Malgré tout, certains étudiants souhaitent s'installer en région ( $22 \%)$.

\section{Plus de la moitié des étudiants envisagent de créer leur entreprise avec un ou des partenaire(s). Et parmi l'ensemble des étudiants, il est à noter que plus du tiers d'entre eux (34\%) accordent de l'importance au développement durable tant au niveau de leurs expériences passées qu'au niveau de la mise en place de leur projet.}

Plus de la moitié des étudiants envisagent de créer leur entreprise avec un ou des parte- naire(s). Et parmi l'ensemble des étudiants, il est à noter que plus du tiers d'entre eux (34\%) accordent de l'importance au développement durable tant au niveau de leurs expériences passées qu'au niveau de la mise en place de leur projet. Aussi, plus du quart d'entre eux ont une idée de projet présentant une dimension sociale (la création d'un centre pour enfants en difficultés, par exemple).

Par ailleurs, seulement $8,5 \%$ des étudiants mentionnent l'existence d'un lien familial dans l'élaboration de leur projet entrepreneurial. Ce lien familial pouvant se traduire par l'aide d'un parent dans l'établissement du réseau de contacts de l'étudiant, par exemple. Finalement, peu de projets concernent la relève de l'entreprise familiale $(3 \%)$.

Toujours en ce qui a trait au projet d'entreprise, plus du tiers des étudiants envisagent le démarrage de leur projet à court terme, soit tout de suite après leurs études, tandis que $17 \%$ d'entre eux entrevoient ce démarrage à moyen terme $(5$ ans après leurs études) et $5 \%$ à long terme (10 ans après l'obtention de leur diplôme).

Toujours en ce qui a trait au projet d'entreprise, plus du tiers des étudiants envisagent le démarrage de leur projet à court terme, soit tout de suite après leurs études, tandis que $17 \%$ d'entre eux entrevoient ce démarrage à moyen terme (5 ans après leurs études) et 5\% à long terme (10 ans après l'obtention de leur diplôme).

Par ailleurs, l'importance qu'accordent les étudiants au travail d'équipe est significative. En effet, plus de $32 \%$ d'entre eux mentionnent l'importance de ce mode de travail collaboratif. Aussi, plusieurs étudiants possèdent un réseau de contacts établi $(27 \%$ ), alors que $32 \%$ soutiennent que ce dernier devrait être davantage développé dans la mise sur pied de leur projet d'entreprise. Finalement, l'importance de la flexibilité ainsi que la conciliation entre le travail et la famille n'est mentionnée que dans $7 \%$ des demandes de bourses. Le tableau 3 présente en détails les données relatives au projet d'entreprise. 
Tableau 3 : Projet d'entreprise

\begin{tabular}{|c|c|c|}
\hline & $\mathbf{N}$ & $\%$ \\
\hline \multicolumn{3}{|l|}{ Type de projet } \\
\hline Entreprise de services & 22 & 37 \\
\hline Commerces & 10 & 17 \\
\hline Artisanat & 1 & 2 \\
\hline Entreprise agro-alimentaire & 7 & 12 \\
\hline Entreprise technologique & 3 & 5 \\
\hline Entreprise touristique & 1 & 2 \\
\hline Autres & 15 & 25 \\
\hline \multicolumn{3}{|l|}{ Forme du projet } \\
\hline Travailleur autonome & 11 & 19 \\
\hline$O B N L$ & 4 & 6 \\
\hline Entreprise incorporée & 37 & 63 \\
\hline Coopérative & 3 & 5 \\
\hline Événement & 1 & 2 \\
\hline Autres & 2 & 3 \\
\hline$N / D$ & 1 & 2 \\
\hline \multicolumn{3}{|l|}{ Lieu de création ville/région } \\
\hline Ville & 21 & 36 \\
\hline Région & 13 & 22 \\
\hline$N / D$ & 25 & 42 \\
\hline \multicolumn{3}{|l|}{ Équipe ou partenariats } \\
\hline $\mathrm{Oui}$ & 33 & 56 \\
\hline Non & 3 & 5 \\
\hline$N / D$ & 23 & 39 \\
\hline \multicolumn{3}{|l|}{ Développement durable } \\
\hline Oui & 20 & 34 \\
\hline $\mathrm{N} / \mathrm{D}$ & 39 & 66 \\
\hline \multicolumn{3}{|l|}{ Dimension sociale } \\
\hline Oui & 15 & 25 \\
\hline Non & 44 & 75 \\
\hline & $\mathbf{N}$ & $\%$ \\
\hline \multicolumn{3}{|l|}{ Lien familial dans construction du projet } \\
\hline Oui & 5 & 9 \\
\hline $\mathrm{N} / \mathrm{D}$ & 54 & 91 \\
\hline \multicolumn{3}{|l|}{ Relève familiale } \\
\hline Oui & 2 & 3 \\
\hline $\begin{array}{r}\mathrm{N} / \mathrm{D} \\
\end{array}$ & 57 & 97 \\
\hline \multicolumn{3}{|l|}{ Création/relève à court, moyen ou long terme } \\
\hline Court terme & 21 & 36 \\
\hline Moyen terme & 10 & 17 \\
\hline Long terme & 3 & 5 \\
\hline N/D & 25 & 42 \\
\hline \multicolumn{3}{|l|}{ Importance du travail d'équipe } \\
\hline Oui & 19 & 32 \\
\hline N/D & 40 & 68 \\
\hline \multicolumn{3}{|l|}{ Réseau de contacts établi } \\
\hline Oui & 16 & 27 \\
\hline Non & 19 & 32 \\
\hline N/D & 24 & 41 \\
\hline Flexibilité/Conciliation travail et famille & & \\
\hline
\end{tabular}




\subsection{Des cheminements propres selon le genre}

Selon les données du tableau 4, quelques éléments distinctifs entre les hommes et les femmes se dégagent. D'abord, davantage d'étudiants que d'étudiantes font état d'une expérience entrepreneuriale dans leurs antécédents, que ce soit la création d'entreprise ou le travail autonome, Environ le tiers des garçons auraient créé une entreprise ou bien auraient été des travailleurs autonomes, contre $15 \%$ de femmes. Les garçons sont aussi plus nombreux à s'être impliqués dans des domaines sportifs que les femmes (50\% d'hommes contre $30 \%$ de femmes). Les femmes sont finalement légèrement plus nombreuses à s'être impliquées au niveau social, communautaire ou environnemental $(63 \%$ des femmes contre $53 \%$ des hommes).

\section{Tableau 4 : Croisement selon le genre}

\begin{tabular}{|c|c|c|}
\hline & \multicolumn{2}{|c|}{ Fréquence $\%(\mathbf{n})$} \\
\hline Expériences entrepreneuriales & Hommes $(n=32)$ & Femmes $(n=27)$ \\
\hline Création d'entreprise ou travailleur autonome & $28(9)$ & $15(4)$ \\
\hline Gestion de projets, organisation d'événements & $19(6)$ & $22(6)$ \\
\hline Emploi ou stage & $53(17)$ & $52(17)$ \\
\hline Implication domaine sportif & $50(16)$ & $30(8)$ \\
\hline Implication sociale, communautaire, environnemental & $53(17)$ & $63(17)$ \\
\hline Activités de leadership & Hommes $(n=22)$ & Femmes $(n=19)$ \\
\hline Présidence de classe, d'association ou de comité & $23(5)$ & $31(6)$ \\
\hline Capitaine d'une équipe sportive & $32(7)$ & $10(2)$ \\
\hline Organisation d'événements, gestion de projets & $45(10)$ & $37(7)$ \\
\hline Emploi ou fonction professionnelle & $0(0)$ & $21(4)$ \\
\hline Projet entrepreneurial & Hommes $(n=32)$ & Femmes $(n=27)$ \\
\hline Entreprise de services & $31(10)$ & $44(12)$ \\
\hline Commerce & $25(8)$ & $8(2)$ \\
\hline Artisanat & $0(0)$ & $4(1)$ \\
\hline Entreprise agro-alimentaire & $12(4)$ & $11(3)$ \\
\hline Entreprise technologique & $9(3)$ & $0(0)$ \\
\hline Entreprise touristique & $3(1)$ & $0(0)$ \\
\hline Autre & $19(6)$ & $33(9)$ \\
\hline Forme du projet & Hommes $(n=32)$ & Femmes $(n=27)$ \\
\hline Travailleur autonome & $6(2)$ & $33(9)$ \\
\hline Organisme à but non lucratif & $9(3)$ & $4(1)$ \\
\hline Entreprise incorporée & $69(22)$ & $55(15)$ \\
\hline Coopérative & $9(3)$ & $0(0)$ \\
\hline Événement & $3(1)$ & $0(0)$ \\
\hline Autre & $3(1)$ & $4(1)$ \\
\hline
\end{tabular}

On constate aussi que les étudiantes sont davantage orientées vers les entreprises de service; elles sont $44 \%$ à choisir ce type d'entreprise. Par ailleurs, les trois projets technologiques répertoriés dans l'échantillon sont attribuables à leurs homologues masculins. De plus, l'ouverture d'un commerce est aussi davantage prisée par les étudiants. Sur les 10 projets de commerces, 8 sont menés par des garçons. Bien qu'une proportion similaire d'étudiantes et d'étudiants affirment avoir occupé des postes de leader, les garçons sont plus nombreux à l'avoir fait dans un contexte sportif (capitaines d'une équipe sportive), avec 
$32 \%$ contre $10 \%$ de femmes. En contrepartie, les filles sont plus nombreuses à avoir occupé des postes de présidence de classe et elles sont les seules à mentionner avoir possédé un emploi ou une fonction professionnelle faisant appel à leurs capacités de leadership.

Un nombre légèrement supérieur d'hommes souhaiteraient démarrer une entreprise incorporée ( $69 \%$ contre $55 \%$ chez les femmes). En outre, les filles semblent davantage attirer par le travail autonome : elles sont 9 sur un total de 27 à envisager cette voie, contre seulement 2 hommes sur un total de 32. Finalement, les trois projets de coopératives sont attribuables aux garçons.

\subsection{L'équipe au cœur des préoccupations}

Une forte proportion des étudiants disent vouloir développer leur projet entrepreneurial en équipe. Or, il semble que ce choix soit particulièrement présent chez les étudiants ayant reçu une formation par projets, laquelle repose en grande partie sur le travail d'équipe. Sur les sept étudiants ayant mentionné avoir profité de ce type d'éducation, cinq mentionnent se lancer en équipe. Par ailleurs, chez les étudiants ayant réalisé des activités de leadership, le choix de créer une entreprise en équipe est prédominant. Comme le montre le tableau 6, l'équipe est particulièrement prisée chez ceux ayant été président de classe ou d'associations et encore ayant organisé des événements.

\section{Une forte proportion des étudiants disent vouloir développer leur projet entrepreneurial en équipe. Or, il semble que ce choix soit particulièrement présent chez les étudiants ayant reçu une formation par projets, laquelle repose en grande partie sur le travail d'équipe.}

L'analyse des données a également permis de constater que plus de la moitié des étudiants ayant voyagé désirent créer leur entreprise en équipe.

Les étudiants optant pour l'entrepreneuriat collectif ou en équipe ont également des intentions de lancer leur projet à plus court terme, comme en fait état le tableau 5. Parmi les 33 projets collectifs identifiés, 15 seraient concrétisés tout de suite après la diplomation des étudiants. Cela pourrait laisser croire que les étudiants souhaitant démarrer leur projet d'entreprise en équipe seraient portés à le faire rapidement Ainsi, l'équipe accélère le processus de réalisation du projet.

Tableau 5 : Croisement selon l'équipe

\begin{tabular}{|c|c|c|c|}
\hline & Oui- Équipe & Non & Information non disponible \\
\hline Terme du projet & & & \\
\hline Court terme & 15 & 1 & 5 \\
Moyen terme & 6 & - & 4 \\
Long terme & 1 & 1 & 13 \\
Information non disponible & 11 & 1 & $\mathbf{2 3}$ \\
Total & $\mathbf{3 3}$ & $\mathbf{3}$ & \\
\hline
\end{tabular}

\subsection{L’importance du réseau}

D'après les données observées et présentées au tableau 6, il est possible de constater que le tiers des étudiants ayant un réseau de contacts établi souhaitent démarrer leur entreprise à court terme, ce qui laisse penser que l'établissement $\mathrm{du}$ réseau de contacts pourrait influencer le terme du projet dans son démarrage. En outre, chez les étudiants désirant créer leur entreprise à long terme, on n'en retrouve aucun ayant un réseau de contacts établi. On remarque donc que plus le terme du projet est long, moins il y a d'étudiants dont le réseau de contacts est établi. 
Tableau 6 : Croisement selon le terme du projet

\begin{tabular}{|c|c|c|c|}
\hline & Court terme & Moyen terme & Long terme \\
\hline Réseau de contacts établi & & & \\
\hline Oui- Établi & 7 & 2 & - \\
Non & 4 & 6 & 3 \\
Information non disponible & 10 & 2 & - \\
Total & $\mathbf{2 1}$ & $\mathbf{1 0}$ & $\mathbf{3}$ \\
\hline
\end{tabular}

\subsection{La ville ou la région?}

Les étudiants envisagent un retour dans la région où ils ont grandi afin de s'y installer et d'y créer leur entreprise, de manière à profiter du réseau et du capital social développé par leurs parents.

Considérant les problématiques d'exode et de dévitalisation des zones rurales, nous nous sommes interrogés sur le lieu d'implantation du projet entrepreneurial envisagé par les étudiants. Selon les données recueillies, plus de la moitié des étudiants possédant au moins un parent entrepreneur souhaiteraient démarrer leur projet d'entreprise en région. Les étudiants envisagent un retour dans la région où ils ont grandi afin de s'y installer et d'y créer leur entreprise, de manière à profiter du réseau et du capital social développé par leurs parents.

Par ailleurs, parmi les 21 étudiants souhaitant démarrer leur entreprise en ville, plus de 17

\section{CONCLUSION}

Cette étude a exploré le thème de l'entrepreneuriat au sein de la génération $\mathrm{Y}$ en cherchant à dresser un portrait des aspirants entrepreneurs de cette génération. Elle a permis de dégager bon nombre de constats intéressants, lesquels sont cohérents avec la littérature existante. En se basant sur les données dégagées de cette étude, certaines grandes caractéristiques telles que l'importance des voyages, du travail d'équipe, la sensibilité au développement durable et la dimension sociale distinguent les membres de la génération $\mathrm{Y}$.

Les résultats appuient ce constat, avec une proportion de $46 \%$ d'étudiants ayant mentionné choisissent l'entreprise incorporée comme forme de projet. Sans être aussi élevé, le nombre d'étudiants souhaitant créer une entreprise incorporée en région est tout de même considérable. En outre, il est pertinent de mentionner que sur les 13 étudiants souhaitant s'installer en région, 5 ont le projet de devenir travailleur autonome.

\subsection{Des entrepreneurs impliqués et écolos}

Le développement durable et l'environnement de même que la dimension sociale constituent des préoccupations très présentes chez les $\mathrm{Y}$. Plus spécifiquement, les données montrent qu'un bon nombre d'étudiants dont le projet présente une dimension sociale ont voyagé. Le tiers des étudiants en sciences et génie, en aménagement, architecture et arts visuels ainsi que le tiers des étudiants en lettres ont des projets présentant une dimension sociale. Aussi, on constate que plus de $60 \%$ des étudiants provenant du programme de sciences et génie et d'agriculture et d'alimentation se disent conscientisés par le développement durable.

avoir effectué des voyages à l'étranger. Il serait important de savoir si les voyages prédisposent à l'entrepreneuriat ou si la relation va plutôt dans l'autre sens. Cependant, il semble approprié pour les universités de favoriser la mobilité internationale des étudiants par le biais de stages ou de programmes d'études à l'étranger. De telles expériences de vie ouvrent les horizons des jeunes et stimulent leur créativité et ouverture des idées nouvelles susceptibles d'être mises en valeur chez eux.

De plus, on peut constater que la tendance à démarrer un projet d'entreprise en équipe est très présente chez les étudiants de la génération $\mathrm{Y}$. 
Dans notre échantillon, $56 \%$ des étudiants ont mentionné souhaiter démarrer leur projet d'entreprise en équipe, un pourcentage beaucoup plus élevé que les $23 \%$ relevés par d'autres études ${ }^{19}$. Cette prédominance des projets collectifs devrait par ailleurs nous amener à questionner le contenu de la formation offerte aux aspirants-entrepreneurs de même que les modes d'accompagnement disponibles. En effet, la plupart des modes d'accompagnement traditionnels (coaching, mentorat ou conseil) reposent sur l'établissement d'une relation dyadique plutôt que de groupe. De plus, comme le travail en équipe et le partenariat constituent une tendance très prisée par les membres de la génération $\mathrm{Y}$ dans la création d'entreprise, les cours sur l'entrepreneuriat devraient traiter les thématiques liées à la gestion des équipes de travail et aux aspects juridiques des différentes formes de partenariat.

Comme le travail en équipe et le partenariat constituent une tendance très prisée par les membres de la génération Y dans la création d'entreprise, les cours sur l'entrepreneuriat devraient traiter les thématiques liées à la gestion des équipes de travail et aux aspects juridiques des différentes formes de partenariat.

Les données concordent avec les écrits stipulant que les membres de la génération $\mathrm{Y}$ seraient davantage conscientisés par l'environnement que les générations précédentes. Ils démontrent aussi un intérêt plus présent de conférer une dimension sociale à leur projet. Encore une fois, ces constats devraient nous inciter à offrir, sur les plans des connaissances et de l'accompagnement, une formation plus adaptée à ce type de projet. Force nous est de constater que pour l'instant, dans les écoles de commerce, l'emphase est nettement mise sur l'entrepreneuriat «d'affaires » par opposition à l'entrepreneuriat social. Les organismes de support aux entrepreneurs se montrent également encore sceptiques lorsqu'un projet à caractère social leur est présenté. Ce scepticisme est encore plus grand chez les bailleurs de fonds.

Une proportion considérable de jeunes semble envisager un retour en région pour y implanter leur projet d'entreprise. Déjà, certains milieux, aux prises avec un problème de dévitalisation et d'exode, misent sur l'entrepreneuriat pour inciter les jeunes à revenir en région après leurs études. Les données semblent montrer que cette avenue pourrait être prometteuse auprès des jeunes nourrissant un projet d'entreprise. L'entrepreneuriat deviendrait ainsi une voie intéressante pour favoriser le retour des jeunes en région, d'autant plus qu'une grande proportion souhaite démarrer son projet d'entreprise à court terme.

\section{Dans les écoles de commerce l'emphase est nettement mise sur l'entrepreneuriat \\ « d'affaires » par opposition à l'entrepreneuriat social. Les organismes de support aux entrepreneurs se montrent également encore sceptiques lorsqu'un projet à caractère social leur est présenté. Ce scepticisme est encore plus grand chez les bailleurs de fonds.}

Bien que cette étude soit de nature fondamentalement exploratoire, elle met en lumière certaines pistes de réflexion. De toute évidence, les aspirants entrepreneurs de la génération $\mathrm{Y}$ ont des besoins et des attentes qui leur sont propres. La façon d'enseigner l'entrepreneuriat et le contenu de cet enseignement doivent être modifiés en conséquence. De plus, les différents intervenants ou parties prenantes qui gravitent autour des jeunes entrepreneurs doivent être conscientisés et adapter leurs pratiques pour mieux répondre aux besoins et aux attentes de ces jeunes. L'enjeu est de taille puisque notre avenir collectif repose en grande partie sur le succès de ces futurs entrepreneurs.

\section{BIBLIOGRAPHIE}

${ }^{1}$ Cennamo, L. et Gardner, D. (2008). Generational Differences in Work Values, Outcomes and PersonOrganisation Values Fit. Journal of Managerial Psychology, 23 (8), 891-906.

${ }^{2}$ Chi, C. G., Gursoy, D. et Maier, T. A. (2008). Generational Differences: An Examination of Work Values and Generational Gaps in the Hospitality Workforce. International Journal of Hospitality Management, 27 (3), 448-458. Campbell, S. M. et Twenge, J. M. (2008). Generational Differences in Psychological Traits and their Impact on the Workplace. Journal of Managerial Psychology, 23 (8), 862-877. 
${ }^{3}$ Alch, M. L. (2008). Get Ready for a New of Worker in the Workplace: the Next Generation. SuperVision, 69 (6), 18-21.

${ }^{4}$ Johnson, J. A. et Lopes, J. (2008). The Intergenerational Workforce, Revisited. Organization Development Journal, 26 (1), 31-36.

${ }^{5}$ Fenn, D. (2008). Cool, Determined and Under 30. Inc., 30 (10), 97-105.

${ }^{6}$ Crampton, S. M. et Hodge, J. W. (2007). Generations in the Workplace: Understanding Age Diversity. The Business Review, 9 (1), 16-22.

${ }^{7}$ Canoë infos, (2008). Quelle est votre génération? http://www2.canoe.com/cgi-bin/imprimer.cgi?id=339688 ${ }^{8}$ Coulon, L., Gardiner, E., Lang, W. et Wong, M. (2008). Generational Differences in Personality and Motivation. Journal of Managerial Psychology, 23 (8), 878-890.

${ }^{9}$ AMP. NATSEM Income and Wealth Report (2007). Generation whY? http://www.amp.com.au/dafiles/ AU\%20Retail/AU\%20Retail/Files/StaticFiles/183327 natsem issue 17 gen_whY.pdf

${ }^{10}$ Hutchings, K., McGuire, D. et Todnem By, R. (2007). Towards a Model of Human Resource Solutions for Achieving Intergenerational Interation in Organisations. Journal of European Industrial Training, 31 (8), 592-608.

11 Turetsky, D. (2006). Generations at Work: New Expectations \& Incentive Requirements. Workspan, 24-27.

${ }^{12}$ Patterson, C. K. (2007). The Impact of Generational Diversity in the Workplace. Diversity Factor, 15 (3), 17-22.

${ }^{13}$ Schwartz, D., Schwartz, T. et Patota, N. (2007). Leveraging Generational Differences for Productivity Gains. Journal of Americain Academy of Business, 11 (2), 1-10.

${ }^{14}$ Cochran, J. (2007). Generation Y Not Now?, Franchising World, 39 (1), 91-94.

15 RBC Groupe Financier, (2007). L'uniformité ne convient pas à tous les entrepreneurs canadiens, selon un récent sondage annuel de RBC. http://www.rbc.com/nouvelles/20070928sme.html

${ }^{16}$ Raising Entrepreneurs, (2009). Gen Y Entrepreneurs- How Do They Measure Up? http://raisingentrepreneurs.org/blog/2009/05/27/genyentrepreneurs-how-do-they-mesure-up

${ }^{17}$ Chain Store Age (2007). Gen Y's Eco-Attitude http://www.chainstoreage.com/search.aspx

${ }^{18}$ Le profil entrepreneurial consiste en un cheminement complémentaire de 12 crédits, intégré aux programmes de baccalauréat. Il permet l'émergence et le développement de compétences entrepreneuriales visant à prendre des initiatives, à concevoir des projets et à les gérer.

${ }^{19}$ Menzies, T. V., Gasse, Y., Diochon, M. et Garand, D. J. (2002). Nascent Entrepreneurs in Canada : An Empirical Study. ICSB 47th World Conference, San Juan, Puerto Rico, Juin.

\author{
Notes \\ ${ }^{a}$ Dans le cas de l'étude, il s'agit de la région de la Capitale \\ Nationale (Ville de Québec et sa périphérie).
}

